Cahiers du MONDE RUSSE

\section{Cahiers du monde russe}

Russie - Empire russe - Union soviétique et États indépendants

\title{
Le paysage russe comme lieu de méditation dans les Récits d'un pèlerin russe
}

Russian nature as a place for meditation in The Way of a Pilgrim

\section{Bernard Marchadier}

\section{(2) OpenEdition}

Journals

\section{Édition électronique}

URL : http://journals.openedition.org/monderusse/9396

DOI : 10.4000/monderusse.9396

ISSN : $1777-5388$

\section{Éditeur}

Éditions de l'EHESS

\section{Édition imprimée}

Date de publication : 15 septembre 2012

Pagination : 479-487

ISSN : $1252-6576$

\section{Référence électronique}

Bernard Marchadier, "Le paysage russe comme lieu de méditation dans les Récits d'un pèlerin russe », Cahiers du monde russe [En ligne], 53/2-3 | 2012, mis en ligne le 01 juillet 2015, Consulté le 01 mai 2019. URL : http://journals.openedition.org/monderusse/9396 ; DOI : 10.4000/monderusse.9396

Ce document a été généré automatiquement le 1 mai 2019. 


\title{
Le paysage russe comme lieu de méditation dans les Récits d'un pèlerin russe
}

\author{
Russian nature as a place for meditation in The Way of a Pilgrim
}

\author{
Bernard Marchadier
}

1 Le livre dont il va être question ici est bien connu du lecteur français. Traduit par Jean Laloy (sous le nom de plume de Jean Gauvain) en 1943 dans les Cahiers du Rhône (Éditions de la Baconnière), présenté par Pierre Pascal dans le $\mathrm{N}^{\circ} 6$ de la revue Dieu vivant (1946), il n'a pas cessé de connaître les rééditions (depuis 1968, aux Éditions du Seuil). Sa renommée est telle qu'il en existe même désormais une adaptation en bande dessinée ${ }^{1}$. Le comédien Michael Lonsdale en donna naguère lecture au cours d'un spectacle organisé dans la crypte de l'église Saint-Sulpice à Paris.

2 Longtemps, les Récits d'un pèlerin ont passé pour un ouvrage anonyme. Il semblerait maintenant établi ${ }^{2}$ qu'ils sont dus à la plume d'un moine russe, le père Mihail Kozlov (1826-1884). L'auteur, issu d'un milieu de vieux-croyants de la région de Smolensk, aurait rejoint les rangs de l'Église synodale officielle à l'âge de vingt ans, à l'époque où il fréquentait le père Matvej Konstantinovskij. Son abandon de la religion de sa famille est peut-être dû aux instances de cet ecclésiastique, connu pour avoir été un prédicateur populaire, charismatique et vigoureux, outre qu'il fut aussi le confesseur de Nikolaj Gogol'. Après avoir pris l'habit, le père Mihail Kozlov sera à son tour, comme le père Matvej, missionnaire auprès des vieux-croyants.

3 Nous le voyons se rendre au Mont Athos, où il s'initie à l'hésychasme et à la prière du cœur. Il retourne en Russie en 1860, et c'est en 1867-1868, lors d'un second séjour à l'Athos, qu'il compose, sur son expérience mystique, un ouvrage qu'il intitule «Iskatel' neprestannoj molitvy » [À la recherche de la prière perpétuelle] ${ }^{3}$. Une copie manuscrite en est envoyée au starets Ambroise du monastère d'Optino (il s'agit de ce même Ambroise que Dostoevskij s'efforcera de peindre dans ses Frères Karamazov sous le nom de Zosime). Ce premier manuscrit est déjà, à peu de choses près, le texte des Récits tel que nous le 
connaissons. Le jugeant utile, Ambroise en recommande la lecture, et les copies de l'ouvrage commencent à circuler à partir de 1870 . C'est en 1881 que le livre paraît à Kazan' sous le titre Otkrovennyj rasskaz strannika duhovnomu svoemu otcu [Récit sincère d'un pèlerin à son père spirituel]. Une deuxième édition paraîtra en 1884, légèrement modifiée par l'évêque Théophane le Reclus (1815-1894), sous son titre définitif, au pluriel: Otkrovennye rasskazy... [Récits sincères...] et c'est celle qui est régulièrement rééditée en français comme Récits d'un pèlerin russe.

Une « suite » des Récits verra le jour en 1911, éditée par la laure de la Trinité-Saint-Serge. Elle est composée d'un cinquième récit et de deux chapitres didactiques sous forme de questions-réponses sur les techniques de prière. Cette partie porte la marque d'une empreinte monastique plus nette et la figure du " pèlerin » s'efface à mesure que le style devient moins alerte et plus édifiant. On s'accorde en général à en attribuer la paternité à l'entourage du starets Ambroise. Elle ne figure pas dans l'édition française courante des Récits".

Quoi qu'il en soit des interventions extérieures qu'a pu subir la première partie des Récits, elle n'en reste pas moins d'une grande qualité formelle, avec une indubitable unité de style. Ce traité de haute spiritualité est aussi un ouvrage littéraire, qui reprend les procédés de la fable classique - les aventures du héros à la recherche du trésor caché - et qui relèverait sans doute par ailleurs du genre picaresque, avec ses récits qui s'emboîtent les uns dans les autres. L'incipit en est exemplaire :

Par la grâce de Dieu je suis homme et chrétien, par actions grand pécheur, par état pèlerin sans abri, de la plus basse condition, toujours errant de lieu en lieu. Pour avoir, j'ai sur le dos un sac avec du pain sec, dans ma blouse la sainte Bible, et c'est tout. Le vingt-quatrième dimanche après la Trinité, j'entrai à l'église pour y prier pendant l'office ; on lisait l'Épître de l'Apôtre aux Thessaloniciens, au passage dans lequel il est dit: Priez sans cesse. Cette parole pénétra profondément dans mon esprit...5

Et l'histoire peut commencer.

7 Dans la traduction française, l'auteur est dit «pèlerin ». Il est difficile effectivement de rendre autrement en français le mot «strannik», mais il faut garder à l'esprit qu'en russe ce vocable suppose l'idée d'errance pieuse, de « vagabondage mystique ». Le « pèlerin » au sens occidental du terme, ce serait plutôt le "bogomolec», celui qui va prier dans les sanctuaires, ou le "palomnik», mot qui renvoie à la « palme » que portaient les pèlerins de Jérusalem (comme l'anglais «palmer» ou le vieux mot français «paumier»). Avant la Révolution, nous dit Pierre Pascal, «la Russie était parcourue de long en large par des milliers de ces errants. À Kiev, le père Mathieu en recevait chaque jour jusqu'à quarante, Tolstoï les a connus et il les aimait, Bounine les a décrits, Chaliapine les a fréquentés.» ${ }^{6}$ Sans doute ces errants donnent-ils une idée de ce que furent chez nous au Moyen-Âge les pèlerins de l'Occident, type qu'illustra encore en France au XVIII ${ }^{e}$ siècle, à un niveau de spiritualité éminent, un saint Benoît Labre. Il est à noter aussi qu'en Russie ce type d'errance ne caractérise pas seulement la paysannerie, et que le rhapsode et pédagogue que fut Grigorij Skovoroda (1722-1794) ou son lointain neveu le philosophe Vladimir Solov'ev (1853-1900) n'eurent jamais de domicile fixe (Skovoroda rédigeant lui-même l'inscription de sa pierre tombale en ces termes : « Le monde a cherché à m'attraper, mais en vain $»^{7}$ ). Tolstoj lui-même mourut en vagabond à la gare d'Astapovo.

Du Pèlerin russe, le lecteur des Récits ignore le nom. Il sait seulement que, né dans la région d'Orel, le Pèlerin est issu d'un milieu simple mais pas pauvre, et qu'il décide de 
partir cheminer lorsque, peu de temps après un incendie qui l'a privé de tous ses biens, sa femme meurt, le laissant inconsolable. Il est très jeune à cette époque puisqu'à la fin du livre, après treize ans d'errance, il a 33 ans (on est en 1859) ${ }^{8}$. Une chute qu'il fit dans son enfance lui démit le bras gauche, qui se dessécha. Impropre du fait de cette infirmité aux travaux de la terre, il put apprendre à lire, ce qui est rare à cette époque dans le monde rural'.

9 Si l'errance est imitation du Christ « qui n'a pas où reposer la tête » (Mat. 8, 20) et mode de vie, elle n'est pas sans but géographique. Toujours le Pèlerin va quelque part, vers quelque lieu saint. Son point de départ n'est pas précisé (néanmoins on devine que c'est en Russie d'Europe), mais on sait vers où il dirige ses pas : Irkutsk en Sibérie pour vénérer les reliques de saint Innocent (1682-1741), puis, via Tobol'sk et Kazan', Odessa pour prendre le bateau qui doit l'emmener en Terre sainte (mais le projet échoue), Kiev pour se recueillir sur les tombeaux des moines des Grottes, Počaev en Ukraine occidentale pour prier la Mère de Dieu. À la fin du dernier récit du second recueil, le Pèlerin part vers l'archipel des Solovki, sur la mer Blanche, pour rejoindre les ermites de l'île d'Anzer, ce " deuxième Athos ». Un coup d'œil sur la carte permet de constater qu'il aura traversé toute la Russie, d'ouest en est et du sud au nord.

La Russie qu'il parcourt (parfois à grandes étapes : plus de soixante-dix kilomètres par jour, mais avec des arrêts qui peuvent se prolonger en fonction des rencontres) est la Russie d'avant l'abolition du servage (1861), la Russie des Récits d'un chasseur de Turgeniev et de Guerre et paix, la Russie rurale des romans de Leskov et des récits de Korolenko, avec les personnages classiques que sont le marchand filou, la jeune fille que l'on veut marier contre son gré, l'aubergiste vieux-croyant, l'ivrogne repenti, le prince devenu mendiant, les grandes maisons où, comme chez la princesse Marija de Guerre et paix, de pieuses gens offrent l'asile aux pèlerins et mendiants, exerçant ainsi la vertu de xénophilie ( strannoljubie), une Russie pleine aussi des dangers de la forêt: les fondrières d'eau glacée, les brigands qui assomment notre Pèlerin, le loup dont il repousse l'attaque à l'aide de son chapelet.

11 La géographie de la Russie qui nous est présentée est aussi anonyme que le Pèlerin. En sa compagnie, on se trouve le plus souvent dans la steppe immense ou en pleine forêt, loin des grandes routes et des villes. Il lui arrive de cheminer plusieurs jours sans rencontrer âme qui vive. Ces déserts humains ne sont pas l'occasion de rêveries herborisantes comme chez le promeneur solitaire, ni de méditations lyriques sur la puissance des éléments comme chez Tjutčev; ils sont encore moins école de perfectionnement moral face à la nature comme chez le Thoreau de Walden. Ils sont avant tout le lieu de l'itinéraire de l'âme à la recherche de Dieu dans le silence et la solitude, le lieu du refuge que les ancêtres vieux-croyants du père Mihail recherchaient dans les forêts d'Outre-Volga et la taïga du Nord ${ }^{10}$. C'est dire si les spectacles du monde et de la nature ne sont pas recherchés. En dehors des grands sanctuaires (qui ne sont jamais dépeints et dont l'auteur ne nous décrit jamais les foules qui s'y pressent ou les miracles qui s'y opèrent) ou des grandes étapes comme Tobol'sk ou Odessa qui sont nommées, on est avec le Pèlerin dans un pays essentiellement sans nom, un pays qui, par le caractère peu différencié, par la non-bigarrure, la pauvreté de ses paysages humains et naturels, semble favoriser l'ascèse du mystique, qui est au départ fuite de la distraction.

Quant à l'État russe, sa présence ne se fait ressentir que par l'obligation faite au Pèlerin de présenter son passeport aux représentants des autorités, sans quoi il serait mis aux fers pour vagabondage. 
13 Le Pèlerin imite le Christ dans son dénuement, et la terre russe qu'il parcourt, parce qu'elle est humble elle aussi, laisse deviner cette Palestine que Jésus a foulée, elle est en quelque sorte icône de la Palestine. À trois reprises, lorsque le Pèlerin évoque son projet d'aller sur les Lieux saints, ceux-ci sont désignés comme "vieille Jérusalem ${ }^{11}$, ce qui donne à penser qu'il se trouve, lui, dans la «nouvelle Jérusalem », autrement dit que la Russie est « nouvel Israël ». Il s'agit là d'un mythe russe très profond. Encore en 1942, le métropolite Sergej de Moscou, face à l'attaque allemande, suppliera le Seigneur «de sauver sa Nouvelle Jérusalem bien aimée, la Sainte Russie, attaquée par les forces païennes $»^{12}$. Au XVII ${ }^{\mathrm{e}}$ siècle, ce mythe avait pris une dimension architecturale grandiose avec le monastère que le patriarche Nikon avait construit à l'image du Saint-Sépulcre non loin de Moscou et qu'il appela "Nouvelle Jérusalem ». L'empereur Constantin, en son temps, avait certes lui aussi voulu faire de ce que les Romains avaient rebâti sous le nom d'« Aelia Capitolina » une "nouvelle Jérusalem », mais il s'agissait seulement pour lui d'axer la cité de David non plus sur le temple juif en ruine mais sur le tombeau du Christ, nouveau centre de la ville elle-même centre du monde ${ }^{13}$. Dans le Saint-Sépulcre constantinien, une urne indiquait l'endroit où se trouvait «l'ombilic du monde ». Elle y est toujours. À partir du patriarche Nikon, auteur d'une véritable translatio Terrae Sanctae, cet ombilic ( " pup zemli») serait désormais en terre russe, devant la réplique du tombeau du Christ ${ }^{14}$. Hâtons-nous toutefois de préciser que si la notion de Russie comme nouvelle Jérusalem n'est pas étrangère à notre Pèlerin, il ne l'utilise pas autrement que comme cliché implicite, et elle n'est en rien essentielle à sa pensée et à sa spiritualité. Il est tout à fait remarquable, à ce titre, qu'à aucun moment les Récits ne mentionnent le mot de «Russie» (pas plus sous la forme Rus' que sous la forme Rossija, qui désigne plus particulièrement l'entité politique).

14 Le paysage russe ressort comme favorable à ce qui motive le pèlerinage et qui n'est autre que la recherche de l'union intime avec Dieu dans la prière intérieure, dite aussi « prière intellectuelle " (umnoe delanie), ou " prière du cœur ». Tout le livre est initiation à cette méthode, sous la direction d'un maître spirituel (celui-là même à qui sont adressés les « récits » du Pèlerin).

15 La méthode spirituelle est définie dans cet ouvrage comme « invocation continuelle et ininterrompue du nom divin de Jésus-Christ par les lèvres, le cœur et l'intelligence, dans la représentation (ou l'imagination : voobraženie) de sa présence en toute occupation, en tout lieu et en tout temps, même pendant le sommeil $\aleph^{15}$. Cette définition est évidemment très importante, et l'auteur et ceux qui ont relu son manuscrit lui ont forcément accordé beaucoup d'attention (elle reste d'ailleurs pratiquement inchangée d'une version à l'autre ${ }^{16)}$. Or il s'y trouve un terme fort étrange, celui «d'imagination» (que le traducteur français esquive bizarrement en parlant de "sentiment de sa présence »). Pourtant le russe dit bien voobraženie (et donc "imagination»), qui évoque si naturellement la « composition de lieu» propre aux Exercices spirituels d'Ignace de Loyola qu'on ne peut manquer d'être surpris ${ }^{17}$. On se souvient en effet que les maitres de la prière du cœur bannissent cette imagination que Simone Weil qualifiera de "combleuse", et qu'en la matière « jamais le novice n'est appelé à se figurer une image extérieure à lui-même » ${ }^{18}$. Il y a sur ce point une latitude étrange chez notre Pèlerin, mais qui l'était peut-être moins en des temps et dans des milieux qui étaient moins systématiques qu'on ne l'a été depuis ${ }^{19}$

Cette invocation perpétuelle $\mathrm{du}$ nom divin s'obtient par des techniques psychophysiologiques qui peuvent paraitre fort mécaniques, et où l'on peut voir une 
amplification du procédé de multiplication des Kyrie eleison pendant les liturgies orientales. La méthode consiste à répéter la formule "Seigneur Jésus Christ, aie pitié de moi » («Gospodi Iisuse Xriste pomiluj mia ») sous la direction d'un maître spirituel. Au XIII siècle Nicéphore l'Hagiorite disait déjà à son disciple: "N'aie d'autre occupation ni méditation que le cri de «Seigneur Jésus Christ, Fils de Dieu, aie pitié de moi ! » Aucune trêve, à aucun prix. Cette pratique, en maintenant ton esprit à l'abri des divagations, le rend imprenable et inaccessible aux suggestions de l'ennemi et, chaque jour, elle l'élève dans l'amour et le désir de Dieu. ${ }^{20}$ Six siècles après, sous d'autres cieux, la méthode n'a pas changé : le starets enjoint au Pèlerin de prononcer la formule 3000 fois par jour (en s'aidant, pour compter, du chapelet orthodoxe, cordelette de laine à 100 ou 150 nœuds), puis 6000 fois, puis 12000 fois, jusqu'à ce que cette prière devienne une seconde nature, se confondant avec la respiration et les battements du cœur, jusqu'à (et c'est très beau) ce qu'un beau matin ce ne soit plus le Pèlerin qui se réveille pour prier mais que ce soit la prière qui le réveille ${ }^{21}$. Il peut alors dire, avec le Cantique des cantiques, « Je dors, mais mon cœur veille » $(5,2)$.

Le maître livre en la matière, dont le Pèlerin se procure pour les deux roubles qui constituent toute sa fortune un exemplaire tout usé, et qui sera avec la Bible héritée de son grand-père le seul imprimé de sa besace, c'est la Philocalie. Le mot signifie " amour de la beauté ", mais d'une beauté toute hellénique, qui se confond avec le bien. Rappelons qu'il s'agit d'un recueil de textes d'une trentaine de saints moines des déserts d'Égypte, du Sinaï et du Mont Athos sur la prière du cœur, établi et publié en 1782 à Venise par Nicodème l'Hagiorite. Cette "prière du cœur ", pratique très ancienne chez les Pères du désert, avait été systématisée en théologie sous le nom d'hésychasme au XIV ${ }^{\mathrm{e}}$ siècle par Grégoire Palamas puis par Nicolas Cabasilas. Elle devint la théologie spirituelle propre à Byzance et à l'orthodoxie orientale lorsque l'adversaire de Palamas, le moine Barlaam de Calabre, de tendance platonisante et nominaliste, fut condamné par un double concile tenu à Constantinople en $1341^{22}$. Les pratiques hésychastes se répandirent en Russie au XV ${ }^{e}$ siècle. Peu de temps après Nicodème l'Hagiorite, le moine russe Paisij Veličkovskij (1722-1794) allait publier en Moldavie une Philocalie (" Dobrotoljubie ») slavonne, enrichie de textes de Palamas absents du recueil de Nicodème. Le livre eut un grand succès, et connut huit éditions ${ }^{23}$. C'est cette Philocalie slavonne que détient le Pèlerin, qui toutefois en donne dans ses Récits des citations non en slavon mais en russe. Puis ce fut en 1877 la monumentale Dobrotoljubie de Théophane le Reclus, qui connut quatre rééditions. L'intelligentsia russe ne découvrit l'hésychasme que dans les années 1860 (comme on le sait, Aleksandr Puškin (1799-1837) et saint Séraphin de Sarov (1760-1833), qui étaient contemporains, n'avaient jamais entendu parler l'un de l'autre). C'est en 1860 en effet que parut à Kiev, sous la plume de l'higoumène Modest, la première étude russe sur Grégoire Palamas, présenté par l'auteur comme héraut de l'anti-occidentalisme ${ }^{24}$. De nos jours, en Russie, l'hésychasme n'est pas seulement une réalité spirituelle dans les monastères; il tend aussi à être une référence nationale chez nombre de penseurs. On citera à ce propos les travaux intéressants d'un Sergej Horuži, qui cherche à établir une anthropologie sur cette base $\mathrm{e}^{25}$.

On ne saurait toutefois associer la "prière du cœur » à une anthropologie spécifique à l'homme russe ou «non occidental». La preuve en est dans le succès ininterrompu dans le public français et francophone de la Petite Philocalie (traduite et présentée par Jean Gouillard) depuis sa parution en 1952 (Éditions des Cahiers du Sud). Un Julien Green notera dans son Journal au 19 décembre 1977: «La Philocalie a été pour moi un compagnon de 
route, le compagnon des heures noires. $»^{26}$ Déjà, saint François de Sales recommande "l'oraison cordiale $»^{27}$. L'Occident chrétien a par ailleurs toujours assidûment pratiqué ce qu'il appelle «l'oraison jaculatoire ", dont il existe de très nombreuses formules, depuis « Domine, Pater et Deus vitae meae » jusqu'à l'exclamation «Mon Dieu et mon tout » que saint François d'Assise répétait du soir au matin. Saint Ignace de Loyola lui-même recommande une méthode proche de celle des moines d'Orient, qu'il appelle «prière par rythme » :

La troisième manière de prier consiste à chaque inspiration ou expiration à prier mentalement en prononçant chaque mot du Pater Noster ou de toute autre prière qu'on récitera en ne prononçant qu'un mot entre l'une et l'autre, on s'attachera surtout à considérer soit le sens de ce mot, soit la personne à qui la prière s'adresse, soit sa propre bassesse, soit la distance qu'il y a entre une telle altesse et une telle bassesse. ${ }^{28}$

19 Certes, la pratique est moins systématique chez saint Ignace que chez les Pères d'Orient, certes aussi il entre dans ce dernier texte de "l'imagination ", mais nous avons vu qu'elle ne semblait pas gêner l'auteur du Pèlerin russe ni lui être étrangère.

Il y a dans le deuxième Récit un moment tout à fait étonnant, c'est celui de la rencontre avec l'intendant polonais employé dans un domaine de Sibérie. Comme toujours avec le Pèlerin, l'entretien en vient vite à porter sur la vie spirituelle et sur la Philocalie. Le Polonais se souvient avoir vu ce livre chez son curé à Wilno, mais il oppose au Pèlerin les arguments qui étaient ceux de Barlaam contre Palamas: il y serait recommandé des pratiques douteuses, une sorte de yoghisme chrétien (Barlaam parlait «d'omphalopsychie»). Or le Pèlerin - qui, notons-le n'a pas hésité à engager une conversation spirituelle avec un "schismatique " voire un " hérétique " latin -, après avoir habilement rappelé que bien des Pères de la Philocalie sont aussi vénérés par l'Église catholique, reprend tous les thèmes de la controverse hésychaste du XIV e siècle pour les exposer au Polonais, qu'il convainc si bien que celui-ci lui demande de lui copier un passage de la Philocalie pour le lire avec son épouse ${ }^{29}$. Quelle liberté, quelle simplicité dans cet épisode !

Rien ne sonne faux en effet dans ce livre, où l'on entend véritablement quelqu'un parler. Il est écrit dans une langue rythmée et naturelle, libre des slavonismes et des formules scolastiques qui encombrent et alourdissent souvent la littérature monastique. Comme il puise par ailleurs au meilleur de la grande tradition ascétique de l'Église, il est libre aussi de la stylisation néo-orthodoxe propre à bien des auteurs du courant slavophile et néoslavophile ${ }^{30}$, libre surtout de ce mythe du « saint moujik» national, cet avatar populiste du mythe de la « Sainte Russie » qu'intellectuels, artistes et politiques russes ont tant fait pour répandre à partir de la seconde moitié du XIXe siècle.

On peut aussi penser que si les Récits du Pèlerin russe - du « Russian Pilgrim », du « Russische Pilger », du «Pellegrino russo »- jouissent depuis des générations et dans tant de pays d'un succès ininterrompu, c'est sans doute parce qu'ils reposent sur une expérience spirituelle et humaine authentique, et non sur des concepts artificiels ou sur un monde rêvé, et parce que c'est un ouvrage non idéologisable, finalement imperméable à la folklorisation. Un grand livre classique qui parle de Dieu à tout homme. 


\section{NOTES}

1. Le Pèlerin russe, scénario et dessin de Gaëtan Evrard, Édition Coccinelle BD.

2. Voir l'étude (en russe) d'I. Basin dans Simvol, N²7, 1992, p. 167-190.

3. Dont on pourra lire le texte dans le numéro de Simvol susmentionné, p. 7-74.

4. Elle est disponible sous le titre : Le Pèlerin russe: Trois récits inédits, P. : Seuil, 1976.

5. Même si l'écho en est sans doute fortuit, le lecteur familier de la littérature russe ne peut s'empêcher de rapprocher cette entrée en matière avec le célèbre commencement des Notes d'un souterrain que Dostoevskij publie au même moment (1864) : "Je suis un homme malade... Je suis un homme méchant. Un homme plutôt repoussant... ». Avec Dostoevskij, le lecteur entre dans la prison de l'ego, quand il s'apprête, avec le Pèlerin, à respirer au grand air du bon Dieu.

6. La religion du peuple russe, Lausanne: L'Âge d'homme, 1973, p. 53. L'historien Vasilij Ključevskij soutenait que les errants constituaient une classe sociale particulière. Voir Michel Evdokimov, Pèlerins russes et vagabonds mystiques, P. : Cerf, 2004, p. 183.

7. « Mir lovil menja no ne poimal».

8. Il est donc né en 1826 et a le même âge que l'auteur, le père Mihail.

9. D'après le prêtre I.S. Belliustin (dans sa lettre du 22 mai 1857 à l'historien Mihail Pogodin), « sur cent paysans de sexe masculin, dix au plus savent lire le Credo et deux ou trois courtes prières [...] Sur un millier d'hommes, il n'y en a pas plus de deux ou trois qui connaissent les Dix Commandements. Quant aux femmes, ce n'est même pas la peine d'en parler. » I.S. Belliustin, Description of the Clergy in Rural Russia, traduite et commentée par G. Freeze, Ithaca - Londres : Cornell University Press, 1985, p. 35.

10. Voir le beau roman de Mel'nikov-Pečerskij qui a pour cadre des communautés de vieux-croyants du XIX ${ }^{\mathrm{e}}$ siècle et qui est précisément intitulé Dans les forêts (P. : Gallimard, 1957). Encore au début des années 1980, des géologues soviétiques découvrirent par hasard une famille de vieux-croyants réfugiés depuis plus de quarante ans dans la forêt sibérienne (voir V. Peskov, Ermites dans la taïga, Arles : Actes Sud, 1992).

11. "Staryj Ierusalim », qu'il convient donc de ne pas traduire, comme dans l'édition française, par « antique Jérusalem » (Récits d'un pèlerin russe, p. 100-111).

12. Cité par F. Stepun, « Moskva - Tretij Rim » in Čaemaja Rossija, SPb., 1999, p. 379.

13. Selon le prophète Ezéchiel. Fidèles à cette conception, les cartes médiévales représentent souvent le monde avec Jérusalem en son centre. Voir à ce sujet la très riche et suggestive étude de W. Hamblin et D. Seely Solomon's Temple: Myth and History, Londres : Thames and Hudson, 2007, notamment p. 90, 101, 106, 109.

14. Voir photographies in G.M. Zelenskaja, Novyj Ierusalim, M., 2003, p. 236-237.

15. Récits, p. 29. J'ai apporté quelques modifications à la traduction, B.M.

16. Voir Simvol, p. 16. 
17. Autre rencontre curieuse, et sans aucun doute significative, entre le Pèlerin et saint Ignace: l'Autobiographie que ce dernier dicta à son secrétaire circula longtemps sous le nom de Récit du pèlerin (voir Saint Ignace de Loyola, Autobiographie, P. : Seuil, 1962, p. 168).

18. Jean Meyendorff, Saint Grégoire Palamas et la mystique orthodoxe, P. : Seuil, 1959, p. 38.

19. Rappelons qu'au $x^{e}{ }^{e}$ siècle une adaptation grecque des Exercices spirituels de saint Ignace due à Nicodème l'Hagiorite était couramment lue au Mont Athos.

20. Ibid., p. 62

21. Récits, p. 16.

22. Meyendorff, Saint Grégoire Palamas et la mystique orthodoxe, p. 98.

23. Jean Gouillard, Petite philocalie de la prière du coeur, P. : Seuil, 1979, p. 12.

24. Ioann Ekonomcev Pravoslavie, Vizantija, Rossija : sbornik statej, M., 1992, p. 168.

25. Voir son article (en russe) «Une anthropologie nouvelle fondée sur une expérience antique ", Voprosy filosofii, $\mathrm{N}^{\circ} 1,2003$.

26. La Terre est si belle, P. : Seuil, 1982, p. 214.

27. Introduction à la vie dévote, P.: Seuil, 1962, p. 65. Sur la pratique de «l'oraison du cœur ", voir aussi le traité du jésuite J.P. de Caussade (1675-1751) (Traité sur l'oraison du cœur, Desclée de Brouwer, s.d., notamment p. 98-99).

28. Cité in Gouillard, Petite philocalie, p. 21.

29. Récits, p. 94-96.

30. Un Serge Nilus, par exemple, qui, dans son recueil Velikoe $v$ malom (1903), enveloppe de considérations d'une piété douceâtre, trouble et maniérée l'étonnante conversation de saint Séraphin de Sarov avec Motovilov qu'il fait connaître au public pour la première fois.

\section{RÉSUMÉS}

Après avoir présenté l'historique de la composition de ce grand classique de la spiritualité russe qui voit le jour dans les années 1880 , l'auteur de cette étude s'attache à situer l'errance spirituelle du Pèlerin à la fois dans l'histoire de la mystique, dans la société et dans le paysage russes. Même s'il apparaît que, pour le Pèlerin, la Russie est bien " Nouvelle Jérusalem » - et donc lieu d'une imitatio Christi - le Pèlerin n'en tire aucune conclusion quant au rôle messianique que, selon les auteurs du courant slavophile et néo-slavophile, elle serait appelée à jouer, et il ne mentionne jamais la «Sainte Russie». Signe sans doute de la profonde authenticité de l'expérience spirituelle du Pèlerin : sa piété n'est guère idéologisable. L'auteur de cette étude voit aussi une preuve de la valeur supranationale de cette mystique dans les rapprochements pertinents qui peuvent être faits avec la spiritualité d'un saint Ignace de Loyola.

Russian nature as a place for meditation in The Way of a Pilgrim

After introducing the background of this great classical book on Russian spirituality composed in the 1880s, the author tries to place the spiritual wanderings of the Pilgrim within the history of Russian mystical thought, society and landscape. Even though it appears that, in the eyes of the 
Pilgrim, Russia is really the "New Jerusalem" and hence the ground for an imitatio Christi, the Pilgrim draws no conclusion as to the messianic role which, according to the Slavophile and neoSlavophile schools of thought, Russia is supposed to play, and he never mentions "Holy Russia." As it does not readily lend itself to being transformed into an ideology, the Pilgrim's spiritual experience bears the marks of authenticity. The author also sees evidence of the supranational value of the Pilgrim's mystical life in the fact that it shares many relevant features with the spirituality of Saint Ignatius Loyola.

\section{AUTEUR}

\section{BERNARD MARCHADIER}

Institut d'études slaves 